\title{
Trophoblastic vasculogenic mimicry in gestational choriocarcinoma
}

\author{
Ie-Ming Shih
}

Departments of Pathology, Oncology and Gynecology and Obstetrics, Johns Hopkins Medical Institutions, Baltimore, MD, USA

\begin{abstract}
Angiogenesis is a characteristic feature of solid tumors, which depend on the newly formed vasculature to prevent hypoxia and to sustain uncontrolled tumor cell proliferation. In this study, we investigated the blood supply system in 10 gestational choriocarcinomas on the basis of histopathological and immunohistochemical features. In all examined cases, morphological analysis demonstrated that blood channels within the center of choriocarcinomas were surrounded by neoplastic trophoblastic cells rather than by endothelial cells. Similarly, there was a lack of CD31 and CD34-positive endothelial cells within choriocarcinomas. In the periphery of choriocarcinomas, tumor cells invaded uterine stroma-derived blood vessels where trophoblastic cells replaced endothelial cells, forming anastomoses between endothelium-lined blood vessels and trophoblastlined pseudovascular channels. Masson's trichrome staining revealed minimal amounts of connective tissue within choriocarcinomas. In contrast, CD31 and CD34-positive blood vessels were present in other types of gestational trophoblastic neoplasms including 8 placental site trophoblastic tumors and 12 epithelioid trophoblastic tumors. These findings provide cogent evidence that choriocarcinoma represents one of a few human tumor types that utilizes vasculogenic mimicry by tumor cell in supporting tumor development. Modern Pathology (2011) 24, 646-652; doi:10.1038/modpathol.2010.231; published online 7 January 2011
\end{abstract}

Keywords: choriocarcinoma; gestational; trophoblastic

Among human carcinomas, gestational trophoblastic neoplasia is unique because it is derived from trophoblastic cells, which are of fetal origin, and thus it represents a semiallograft in patients. Gestational trophoblastic neoplasia includes choriocarcinoma, placental site trophoblastic tumor, and epithelioid trophoblastic tumor. ${ }^{1,2}$ Among these, choriocarcinoma is the most common and, if untreated, is clinically aggressive, and is associated with the development of widespread metastasis and high mortality. ${ }^{3}$ Choriocarcinoma frequently follows a complete hydatidiform mole which is characterized by exaggerated trophoblastic hyperplasia. ${ }^{4}$ Previous morphological and immunohistochemical studies have suggested that choriocarcinoma develops as a result of neoplastic transformation of trophoblastic stem cells, presumably the cytotrophoblast. $^{1,5}$ The neoplastic cytotrophoblastic cells, like normal cytotrophoblasts on the villous surface

Correspondence: Professor I-M Shih, MD, PhD, Departments of Pathology, Oncology and Gynecology and Obstetrics, Johns Hopkins Medical Institutions, CRB-2, 1550 Orleans Street, Baltimore, MD 21231, USA. E-mail: ishih@jhmi.edu

Received 19 October 2010; revised 11 November 2010; accepted 11 November 2010; published online 7 January 2011 of early placentas, differentiate into either extravillous (intermediate) trophoblast responsible for establishing the implantation site, or differentiate into syncytiotrophoblast which functions as a hormonal organ and regulates molecular exchange across the fetal-maternal interface. As a result, choriocarcinoma is morphologically composed of variable amounts of neoplastic cytotrophoblast, syncytiotrophoblast, and extravillous (intermediate) trophoblast, which, in aggregate, comprise the characteristic features of the 'biphasic' pattern (mononucleate trophoblast alternating with syncytiotrophoblast) of choriocarcinoma.

In recent years, progress has been made toward elucidating the biology of human trophoblast including the differentiation of distinct trophoblastic sub-populations and their physiological integration during development. ${ }^{6-8}$ The identification and characterization of the genes expressed in human trophoblast have enhanced our understanding of the lineage and differentiation program of trophoblast and its relationship to trophoblastic tumors and tumor-like lesions. ${ }^{9}$ Molecularly, several genes and pathways have been reported to participate in the pathogenesis of choriocarcinoma. These include epidermal growth factor (EGF) receptor, ${ }^{10}$ 
DOC-2/hDab2 (a candidate tumor suppressor gene), ${ }^{11}$ the ras GTPase activating protein,${ }^{12} \mathrm{C}$-myc, c-erb-2, c-fms, and bcl-2 oncoproteins. ${ }^{13}$ It has been demonstrated that trophoblastic cells in normal placentas and gestational trophoblastic neoplasia upregulate HLA-G expression. In fact, among human cancers analyzed, gestational trophoblastic neoplasia-including choriocarcinoma-contains the highest levels of HLA-G expression. ${ }^{14}$ A plausible hypothesis is that HLA-G expressed by choriocarcinomas assists the escape of tumor cells from host immune recognition and promotes tumor growth. ${ }^{15}$

In normal placenta, syncytiotrophoblast covering the surface of chorionic villi has an important role in molecular exchange, including oxygen exchange, across the maternal and fetal interface. The endothelial cell-like functions of syncytiotrophoblast suggest that in choriocarcinoma, in which syncytiotrophoblast is abundantly present, syncytiotrophoblast may be responsible for blood supply and circulation. Thus, trophoblastic cells likely substitute for angiogenesis in the development of choriocarcinoma. To test this interesting hypothesis, we conducted a morphological and immunohistochemical study on gestational choriocarcinomas of the uterus. For comparison, we also examined other gestational trophoblastic neoplasia including placental site trophoblastic tumor and epithelioid trophoblastic tumor, which lack the syncytiotrophoblastic component.

\section{Materials and methods}

\section{Tissue Material}

Paraffin-embedded tissues of uterine choriocarcinomas were collected in the Department of Pathology, the Johns Hopkins Hospital, Maryland, and National Taiwan University Hospital. All cases were anonymous and were obtained from patients in accordance with the guidelines of the institutional review board. We selected 10 choriocarcinomas on the basis
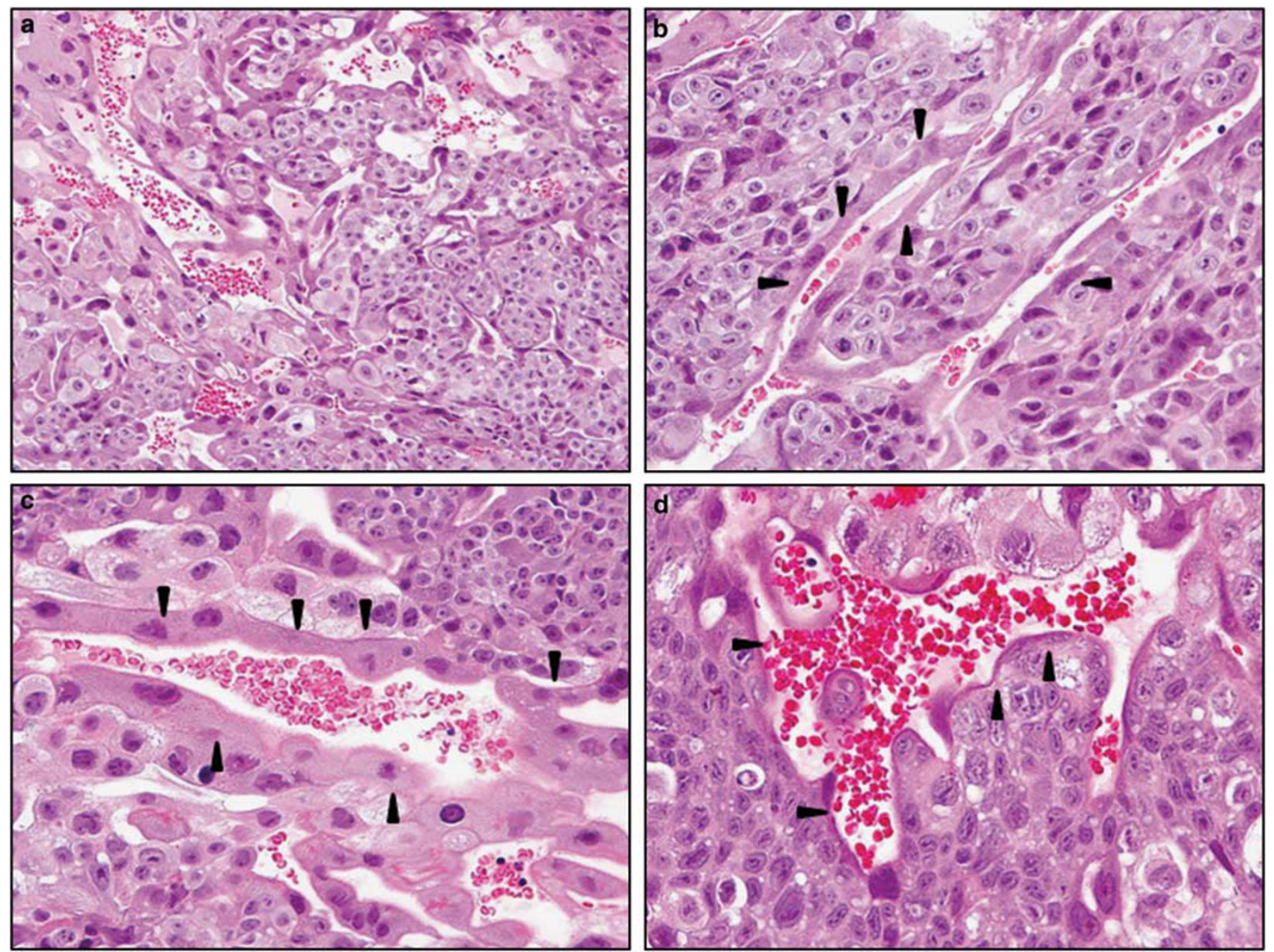

Figure 1 Photomicrographs of choriocarcinomas (hematoxylin and eosin stain). (a) At low magnification, blood lakes or channels are haphazardly dispersed within the choriocarcinoma. (b) Medium magnification shows pseudovascular channels surrounded by syncytiotrophoblast (arrowheads). Red blood cells are present in the lumen. (c, d) Higher magnification views of two different choriocarcinomas. The blood channels or lakes are lined by syncytiotrophoblast (arrowheads), which surrounds mononucleate trophoblastic cells. No morphological evidence of endothelial cells is detected. 
of the presence of at least one tumor area showing a sizable lesion that would allow assessing the presence of vascular channels in the center of the tumor. Cases with small fragments of choriocarcinoma or with massive hemorrhage and necrosis were not included because of the concern that these tumors would only contain the periphery of the choriocarcinoma, and the center of the tumor would not be available for study. Two blocks were studied in each of five choriocarcinomas and one block was available to study in the remaining five cases. In addition, we also identified 12 placental site trophoblastic tumors and 10 epithelioid trophoblastic tumors that were included in this study. Hematoxylin and eosin (H\&E)-stained sections were prepared from all cases for histopathological examination using the standard protocol.

\section{Immunohistochemistry and Masson's Trichrome Staining}

We used CD31/PECAM-1 and CD34 as markers for endothelial cells. Immunohistochemistry for CD31 and CD34 was performed using a Ventana Benchmark device with provided Ventana kits (Ventana, Tucson, AZ, USA) following the standard protocol. A positive reaction was detected by development with 3,3'-diaminobenzidine. Masson's trichrome staining was used to identify connective tissues in choriocarcinoma.

\section{Tumor Xenografts in Mice}

Both JEG3 and JAR choriocarcinoma cell lines were obtained from American Tissue Collection Center (Rockville, MD, USA). The cells were cultured in RPMI1640 supplemented with 5\% fetal bovine serum. Cells grown to a subconfluent density were harvested and were injected into the subcutaneous tissue of athymic $\mathrm{nu} / \mathrm{nu}$ mice $\left(5 \times 10^{6}\right.$ cells per injection, 5 mice per cell line). Two weeks after tumor injection, tumor xenografts were excised, fixed in formalin, and H\&E sections were prepared for histological examination.

\section{Results}

Based on H\&E staining, we observed that the spaces filled with red blood cells within the center of a choriocarcinoma were completely surrounded by trophoblastic cells, most commonly by syncytiotrophoblasts (Figure 1). There was no morphological evidence of endothelial cells surrounding the blood space. This finding was observed in all 10 choriocarcinomas examined. In contrast, endothelium-lined
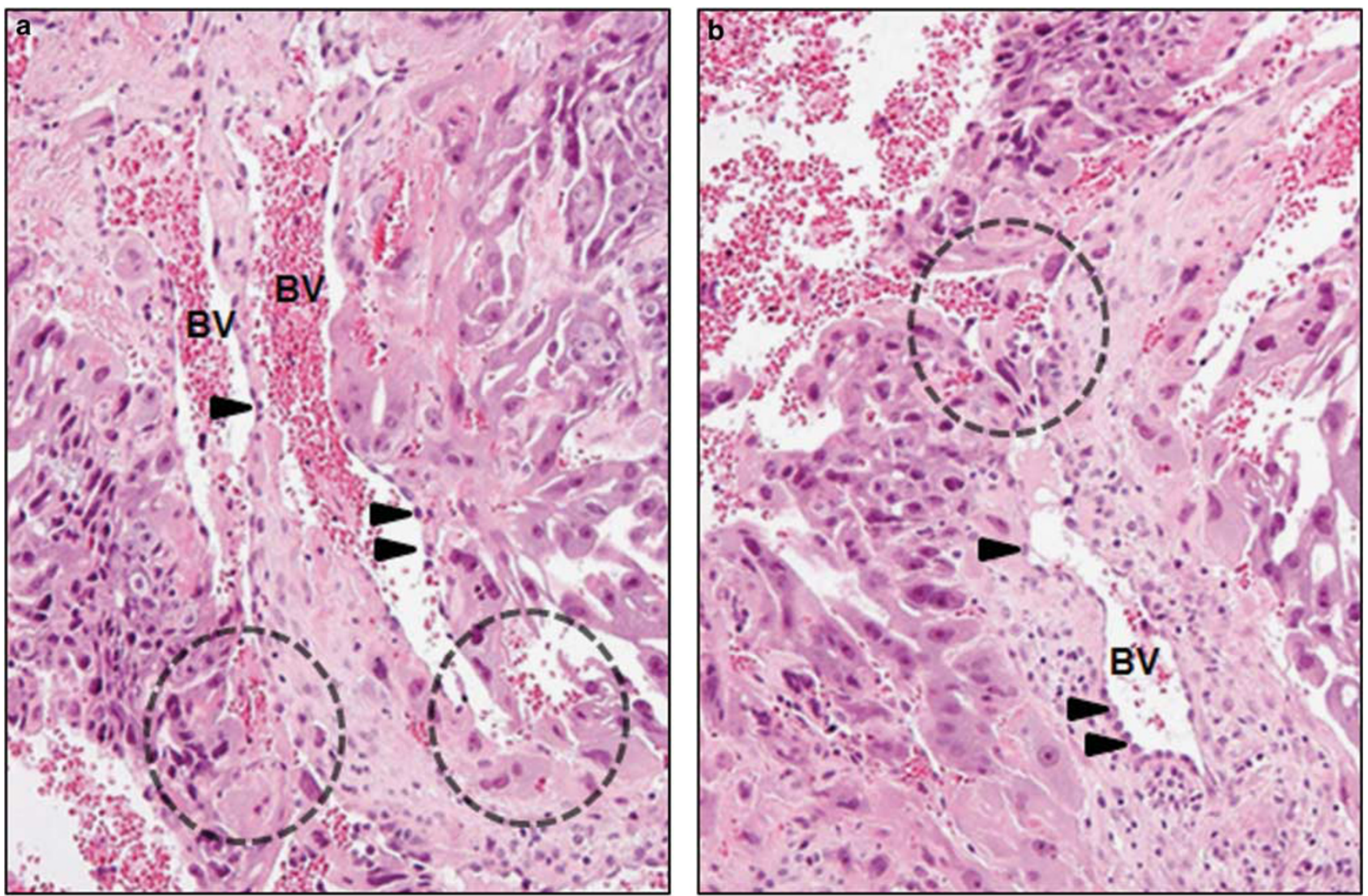

Figure 2 Vascular channels in the border of a choriocarcinoma. (a, b) Blood vessels (BV) lined by endothelial cells (arrowheads) are identified outside the tumor and are in direct communication with choriocarcinoma (circles). 
vascular channels were detected in the uterine stroma outside the tumor. A careful examination of the border of choriocarcinomas demonstrated an intimate anastomosis between endothelium-lined and trophoblast-lined blood channels (Figure 2). This morphological finding suggested the possibility that the center of choriocarcinomas was devoid of new blood vessel formation as tumor developed. Therefore, we stained all cases with antibodies against two well-established endothelial cell markers, CD31 and CD34, and stained five representative cases with Masson's trichrome stain to detect connective tissue. We found that CD31 and CD34-positive blood vessels were present outside the tumor and in the periphery of the tumor mass (Figure 3). Similarly, connective tissue was only detected outside the choriocarcinoma (Figure 3). At higher magnification, choriocarcinoma cells which had invaded into CD31 and CD34-positive blood vessels and which had partially replaced the endothelial cells at the margins of the tumors could be seen (Figure 4). In contrast to the findings in choriocarcinoma, endothelium-lined CD31 and CD34-positive blood vessels were found in the center of tumor nodules of all placental site trophoblastic tumors and epithelioid trophoblastic tumors that were examined (Figure 5).
We next injected JEG3 and JAR choriocarcinoma cells into the subcutaneous tissues of immunocompromised $n u / n u$ mice to determine whether a similar histological pattern occurred in human choriocarcinoma xenografts. Both choriocarcinoma cell lines produced tumors within 1 week. Histological examination of the xenografts revealed poorly differentiated tumor mass without discernable trophoblastic differentiation (data not shown). In particular, there was no evidence of syncytiotrophoblastic cells. The tumors were highly angiogenic as intratumoral blood vessels could be easily detected throughout the tumor in all specimens. Thus, the histopathological features of choriocarcinoma xenografts were strikingly different from the histopathological features found in surgical specimens from choriocarcinoma patients.

\section{Discussion}

The results presented above have several biological implications in studying choriocarcinoma. In human solid tumors, lack of angiogenesis is a very rare event and was initially reported in melanomas and occasionally in other non-melanoma cancers including breast, ovarian, prostate, and renal clear cell
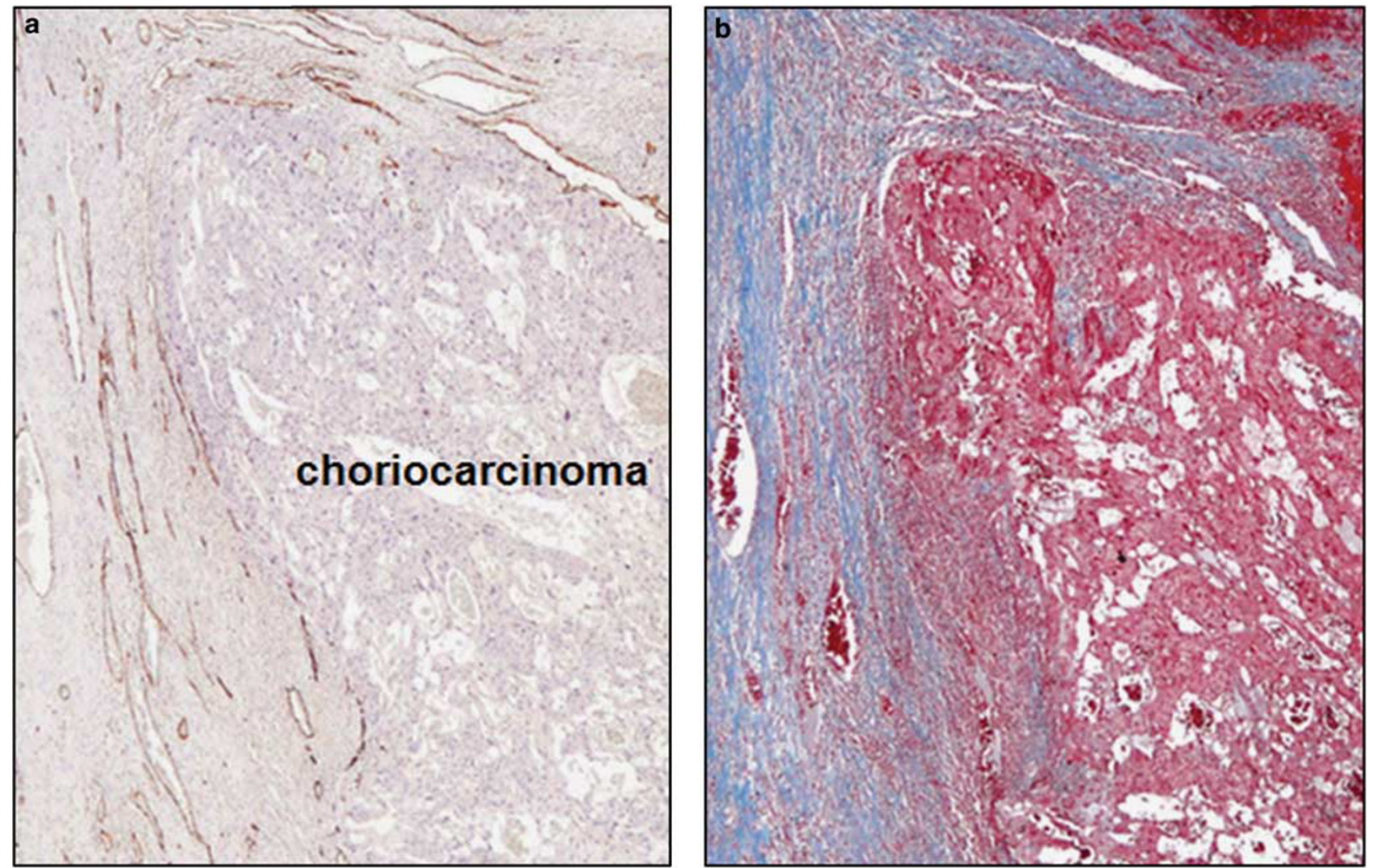

Figure 3 View of a uterine choriocarcinoma showing the junction between the choriocarcinoma and normal uterine tissue (low magnification). (a) CD31-stained section demonstrates CD31-positive blood vessels outside the choriocarcinoma whereas there is no evidence of CD31 immunoreactivity inside the tumor. (b) Masson's trichrome stain shows abundant connective tissue (blue) in the uterine tissue, but little within the choriocarcinoma. The red staining indicates cellular components. 

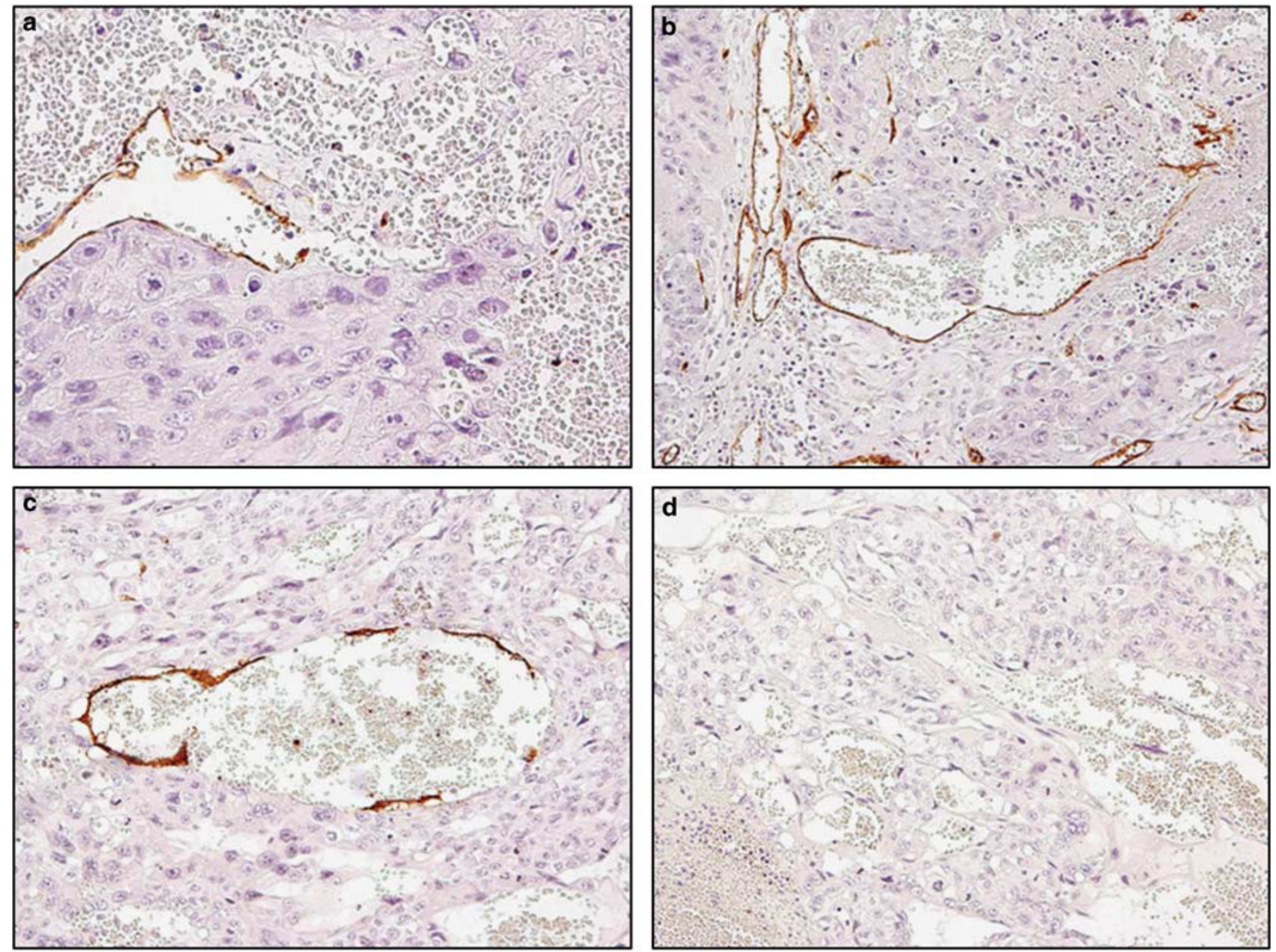

Figure 4 CD31 immunostaining on choriocarcinomas. (a-c) Blood vessels are infiltrated by choriocarcinoma cells and the CD31-positive endothelial cells are partially replaced by tumor cells. (d) CD31 staining fails to detect CD31-positive endothelial cells in the center of a choriocarcinoma.

carcinomas. ${ }^{16}$ Blood circulation was established in those tumors by creating vascular channels lined by tumor cells, and this process has been termed as 'vasculogenic mimicry', which underscores the de novo generation of vascular channels without involvement of non-neoplastic endothelial cells. ${ }^{17,18}$ With vasculogenic mimicry, it is thought that tumor cells are able to sustain tumor growth and, to some extent, prevent tumor hypoxia. Thus, choriocarcinoma represents another example of human neoplastic diseases featuring vasculogenic mimicry, but unlike other tumor types, vasculogenic mimicry in choriocarcinoma occurs in all cases examined, indicating that vasculogenic mimicry is a general phenomenon associated with choriocarcinoma.

In this study, we found that the tumor-formed pseudovascular channels in choriocarcinomas were contiguous with the endothelial cell-lined blood vessels surrounding the tumor mass, suggesting that choriocarcinoma cells invade adjacent blood vessels and drain blood into the intercellular space within choriocarcinoma. Indeed, the vascular channels lined by both endothelial and trophoblastic cells were clearly visible in the tumor-uterine interface. As choriocarcinoma did not contain a substantial amount of connective tissue as evidenced by the Masson's trichrome stain, it can be concluded that choriocarcinoma lacks underlying matrix support and it is possible that the blood flow from uterine vessels creates haphazard labyrinth-like pseudovascular channels. The observation that the pseudovascular channels were lined in almost all cases by syncytiotrophoblast rather than cytotrophoblast and extravillous (intermediate) trophoblast is reminiscent of trophoblastic ingrowth into endometrium following blastocyst implantation, in which syncytiotrophoblast located at the implantation pole encapsulates cytotrophoblast and directly invades into the implantation site. ${ }^{19}$ The capacity of syncytiotrophoblast to perform endothelium-like functions such as controlling substrate exchange across the villous surface has been established. ${ }^{20,21}$ Thus, choriocarcinoma assumes the physiology of normal trophoblast and operates its blood supply through tumor syncytiotrophoblast-formed blood vessels. To assess whether syncytiotrophoblast is 

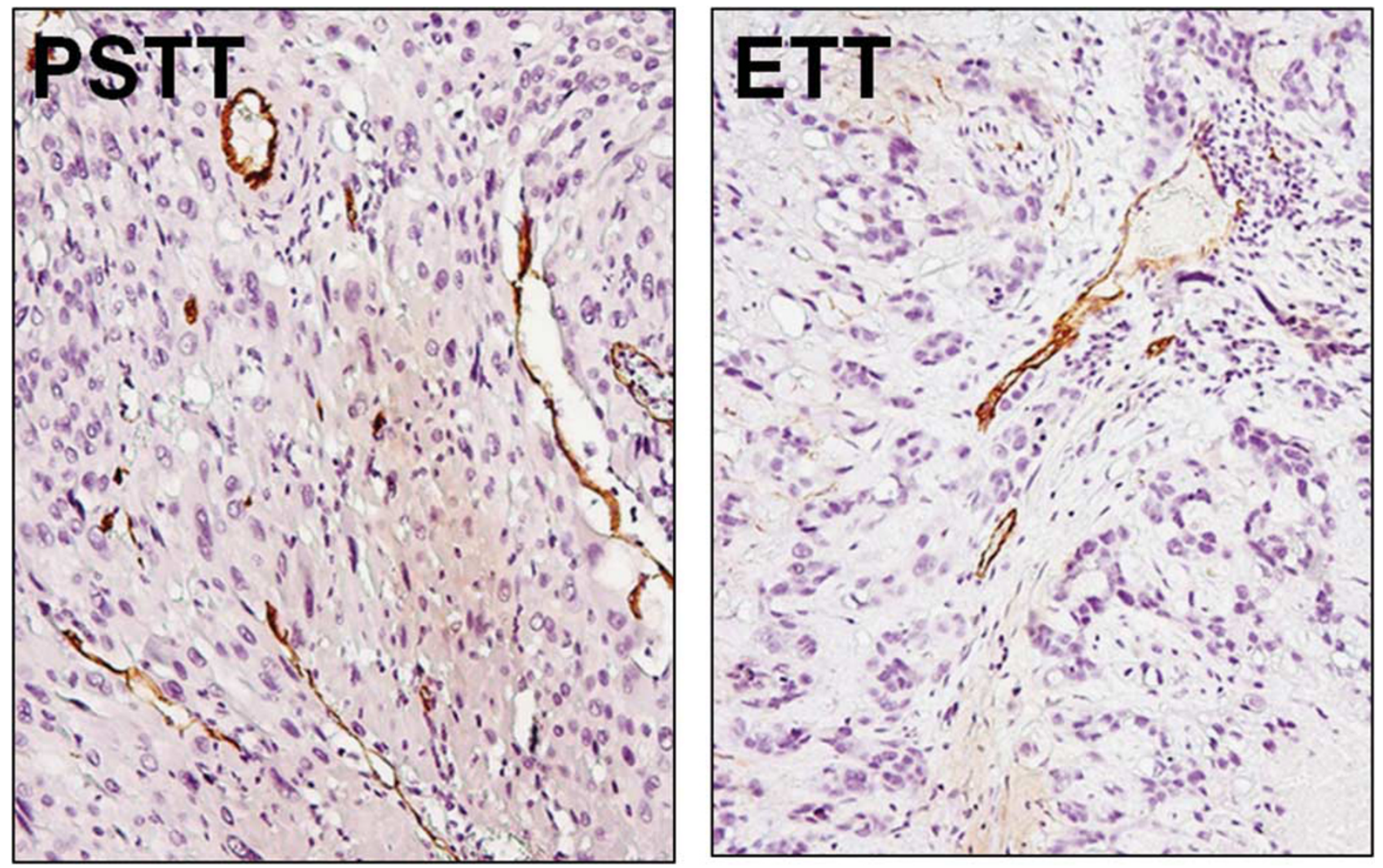

Figure 5 The presence of CD31-positive blood vessels (brown staining) in representative placental site trophoblastic tumor (PSTT) and epithelioid trophoblastic tumor (ETT). Both types of gestational trophoblastic neoplasia show scattered blood vessels inside the tumors. Connective tissue stroma is also detected in the tumors.

critically associated with pseudovascularization, we also analyzed other gestational trophoblastic neoplasia including placental site trophoblastic tumor and epithelioid trophoblastic tumor because in these tumors the trophoblastic cells are of intermediate trophoblastic type and, unlike cytotrophoblastic cells, do not generally differentiate into syncytiotrophoblast. ${ }^{1,22}$ Our finding that CD31 and CD34-positive blood vessels were present in placental site trophoblastic tumor and epithelioid trophoblastic tumor indicates that angiogenesis occurs in both types of gestational trophoblastic neoplasia and supports the view that choriocarcinoma is different from placental site trophoblastic tumor and epithelioid trophoblastic tumor. Moreover, although originally derived from choriocarcinomas, both JEG3 and JAR tumor xenografts in mice did not exhibit any morphological features of human choriocarcinoma. Choriocarcinoma xenografts were composed exclusively of undifferentiated tumor cells without differentiation into syncytiotrophoblast. Moreover, there was remarkable angiogenesis in those tumor xenografts. These findings suggest that syncytiotrophoblast may facilitate the formation of vasculogenic mimicry in choriocarcinoma.

It is not known whether the absence of angiogenesis in choriocarcinoma is a result or a cause of the lack of connective tissues in choriocarcinoma.
However, one plausible hypothesis is that tumor stroma is not generated during the development of choriocarcinoma because vasculogenic mimicry by neoplastic trophoblastic cells is sufficient to support the growth of the choriocarcinoma. Alternatively, the rapid and expansile growth of choriocarcinoma may not allow for the typical infiltrative pattern of spread into connective tissue as seen in many other neoplasms. Nevertheless, the blood and oxygen supply in choriocarcinoma may not be as effective as the supply provided through true angiogenesis as occurs in other solid tumors. Without the support of connective tissue, the trophoblastic cell-lined vascular space may be prone to collapse as a result of expansible growth or necrosis of tumor cells compromising local blood circulation. Thus, tumor cells in a choriocarcinoma are more vulnerable to ischemic changes that frequently result in massive tumor necrosis and hemorrhage, which are the main histopathological features that characterize most choriocarcinomas and which have been used as diagnostic criteria for this tumor. ${ }^{22}$ In fact, it is not unusual to encounter a choriocarcinoma with a substantial amount of hemorrhage and necrosis in the center and only a few layers of viable tumor cells located in the tumor margins.

In conclusion, this study provides evidence that choriocarcinoma lacks new blood vessel formation in the center of the tumor. Rather, a choriocarcinoma 
establishes its own intricate pseudovascular networks that are lined by syncytiotrophoblastic cells rather than by endothelial cells. It remains to be determined why angiogenesis fails to occur during choriocarcinoma evolution. It would be interesting to determine whether syncytiotrophoblastic differentiation in choriocarcinoma is required for vasculogenic mimicry. Finally, the utilization of vasculogenic mimicry, which is less effective than bona fide angiogenesis, can also explain the massive hemorrhage and necrosis that are frequently associated with choriocarcinoma.

\section{Acknowledgements}

I am grateful for the tissue materials contributed by Dr Robert J Kurman (Johns Hopkins Hospital) and Dr Tsui-Lien Mao (National Taiwan University Hospital). I also appreciate the effort of Dr Stefanie Ueda who performed the choriocarcinoma xenografts in nude mice.

\section{Disclosure/Conflict of interest}

The author declares no conflict of interest.

\section{References}

1 Shih IM. Gestational trophoblastic neoplasia-pathogenesis and potential therapeutic targets. Lancet Oncol 2007;8:642-650.

2 Wells M. The pathology of gestational trophoblastic disease: recent advances. Pathology 2007;39:88-96.

3 Seckl MJ, Sebire NJ, Berkowitz RS. Gestational trophoblastic disease. Lancet 2010;376:717-729.

4 Cheung AN, Zhang HJ, Xue WC, et al. Pathogenesis of choriocarcinoma: clinical, genetic and stem cell perspectives. Future Oncol 2009;5:217-231.

5 Mao TL, Kurman RJ, Huang CC, et al. Immunohistochemistry of choriocarcinoma: an aid in differential diagnosis and in elucidating pathogenesis. Am J Surg Pathol 2007;31:1726-1732.

6 Maltepe E, Bakardjiev AI, Fisher SJ. The placenta: transcriptional, epigenetic, and physiological integration during development. J Clin Invest 2010;120:1016-1025.

7 Weier JF, Weier HU, Jung CJ, et al. Human cytotrophoblasts acquire aneuploidies as they differentiate to an invasive phenotype. Dev Biol 2005;279:420-432.
8 Red-Horse K, Zhou Y, Genbacev O, et al. Trophoblast differentiation during embryo implantation and formation of the maternal-fetal interface. J Clin Invest 2004;114:744-754.

9 Shih I-M. Trophogram, an immunohistochemistrybased algorithmic approach, in the differential diagnosis of trophoblastic tumors and tumorlike lesions. Ann Diagn Pathol 2007;11:228-234.

10 Tuncer ZS, Vegh GL, Fulop V, et al. Expression of epidermal growth factor receptor-related family products in gestational trophoblastic diseases and normal placenta and its relationship with development of postmolar tumor. Gynecol Oncol 2000;77: 389-393.

11 Fulop V, Mok SC, Berkowitz RS. Molecular biology of gestational trophoblastic neoplasia: a review. J Reprod Med 2004;49:415-422.

12 Stahle-Backdhal M, Inoue M, Zedenius J, et al. Decreased expression of Ras GTPase activating protein in human trophoblastic tumors. Am J Pathol 1995; 146:1073-1078.

13 Fulop V, Mok SC, Genest DR, et al. c-myc, c-erbB-2, c-fms and bcl-2 oncoproteins. Expression in normal placenta, partial and complete mole, and choriocarcinoma. J Reprod Med 1998;43:101-110.

14 Shih Ie M. Application of human leukocyte antigen-g expression in the diagnosis of human cancer. Hum Immunol 2007;68:272-276.

15 Singer G, Kurman RJ, McMaster M, et al. HLA-G immunoreactivity is specific for intermediate trophoblast in gestational trophoblastic disease and can serve as a useful marker in differential diagnosis. Am J Surg Pathol 2002;26:914-920.

16 Paulis YW, Soetekouw PM, Verheul HM, et al. Signalling pathways in vasculogenic mimicry. Biochim Biophys Acta 2010;1806:18-28.

17 Maniotis AJ, Folberg R, Hess A, et al. Vascular channel formation by human melanoma cells in vivo and in vitro: vasculogenic mimicry. Am J Pathol 1999;155: 739-752.

18 Folberg R, Hendrix MJ, Maniotis AJ. Vasculogenic mimicry and tumor angiogenesis. Am J Pathol 2000; 156:361-381.

19 Benirschke K, Kaufmann P (eds). Early Development of the Human Placenta. In: Pathology of the human Placenta. 3rd edn., Springer-Verlag: New York, 1995.

20 Sibley CP. Understanding placental nutrient transferwhy bother? New biomarkers of fetal growth. J Physiol 2009;587:3431-3440.

21 Moe AJ. Placental amino acid transport. Am J Physiol 1995;268:C1321-C1331.

22 Shih IM, Kurman RJ. The pathology of intermediate trophoblastic tumors and tumor-like lesions. Int J Gynecol Pathol 2001;20:31-47. 